University of Nebraska - Lincoln

DigitalCommons@University of Nebraska - Lincoln

8-1-1997

\title{
Magnetism in one dimension: Fe on $\mathrm{Cu}(111)$
}

J. Shen

Max-Planck-Institut für Mikrostrukturphysik, shenj5494@fudan.edu.cn

Ralph Skomski

University of Nebraska-Lincoln, rskomski2@unl.edu

M. Klaua

Max-Planck-Institut für Mikrostrukturphysik

H. Jenniches

Max-Planck-Institut für Mikrostrukturphysik

S. Sundar Manoharan

Max-Planck-Institut für Mikrostrukturphysik

See next page for additional authors

Follow this and additional works at: https://digitalcommons.unl.edu/physicsskomski

Part of the Physics Commons

Shen, J.; Skomski, Ralph; Klaua, M.; Jenniches, H.; Manoharan, S. Sundar; and Kirschner, J., "Magnetism in one dimension: Fe on $\mathrm{Cu}(111) "$ (1997). Ralph Skomski Publications. 21.

https://digitalcommons.unl.edu/physicsskomski/21

This Article is brought to you for free and open access by the Research Papers in Physics and Astronomy at DigitalCommons@University of Nebraska - Lincoln. It has been accepted for inclusion in Ralph Skomski Publications by an authorized administrator of DigitalCommons@University of Nebraska - Lincoln. 


\section{Authors}

J. Shen, Ralph Skomski, M. Klaua, H. Jenniches, S. Sundar Manoharan, and J. Kirschner

This article is available at DigitalCommons@University of Nebraska - Lincoln: https://digitalcommons.unl.edu/ physicsskomski/21 


\title{
Magnetism in one dimension: $\mathrm{Fe}$ on $\mathrm{Cu}(111)$
}

\author{
J. Shen, ${ }^{*}$ R. Skomski, M. Klaua, H. Jenniches, S. Sundar Manoharan, and J. Kirschner \\ Max-Planck-Institut für Mikrostrukturphysik, Weinberg 2, 06120 Halle, Germany
}

(Received 21 April 1997)

\begin{abstract}
The magnetism and the morphology of one-dimensional $\mathrm{Fe}$ stripes on a $\mathrm{Cu}(111)$ vicinal surface with perpendicular magnetization are investigated. Scanning tunneling microscopy shows that the Fe stripes have nanometer width and are aligned in parallel along the [011] direction. From the magnetization curves it is inferred that the stripes exhibit a ferromagnetic behavior well below the nominal thickness of one monolayer. In contrast to two-dimensional films, the magnetization of the stripes is not only temperature but also time dependent. The dynamics of the stripes have been described by a micromagnetic Ising model with Glauber dynamics, yielding an effective anisotropy constant $K_{1}$ of $0.65 \pm 0.15 \mathrm{M} \mathrm{J} / \mathrm{m}^{3}$. [S0163-1829(97)01230-7]
\end{abstract}

The dimensionality of magnetic matter has a strong influence on its magnetic properties. Both theory ${ }^{1}$ and experiment ${ }^{2}$ indicate that in a two-dimensional magnetic system long-range ferromagnetic order prevails at nonzero temperature, once anisotropies are present. By contrast, in a onedimensional (1D) magnetic system, theoretical arguments show that even a highly anisotropic system does not yield a zero-field equilibrium spontaneous magnetization. ${ }^{3}$ The prototype model is the Ising chain of localized spins with nearest-neighbor exchange interaction, which has no longrange order at nonzero temperature. ${ }^{4}$ Experimentally 1D magnetism on compound material has been extensively studied from the $1970 \mathrm{~s}^{5}$ For $3 d$ metals, a possible experimental realization of a 1D system is an array of a large number of parallel, nanometer-wide magnetic stripes on nonmagnetic substrates. In a pioneering experiment Elmers et al. ${ }^{6}$ produced Fe stripes by step-flow growth on a W(110) crystal. These stripes showed a strong in-plane anisotropy along the stripe axis and a two-dimensional Ising-like behavior, despite their quasi-1D appearance. In our experiment Fe stripes of $1-2$ atom height by $5-15$ atom width are produced by stepedge decoration of a stepped $\mathrm{Cu}$ crystal (see Fig. 1). Because of their perpendicular magnetization axis and their high aspect ratio between cross section and length, the Fe stripes come close to an array of (finite) Ising chains. Yet, these quasi-1D stripes show all the usual signs of ferromagnetism: hysteresis loops and a critical temperature, above which the magnetization disappears. We will show below that this apparent conflict is resolved by a statement put forward by Jacobs and Bean ${ }^{7}$ in 1963: “ . . . the one-dimensional Ising chain (or any anisotropic chain) is not ferromagnetic in equilibrium but will, in fact, show all the usual characteristics of ferromagnetic matter owing to the difficulty of reaching equilibrium.' It is only today, several decades later, that the experimental means and tools have become available to test the validity of their predictions on $3 d$ pure metals.

The experiments were performed in a multichamber system including a molecular beam epitaxy (MBE) preparation chamber, a scanning tunneling microscopy (STM) chamber, an analysis chamber equipped with cylindrical mirror analyzer (CMA) based Auger electron spectroscopy (AES) and low-energy electron diffraction (LEED), and a magnetooptical Kerr effect (MOKE) chamber. The base pressure of the individual chambers is better than $5 \times 10^{-11}$ mbar. The characteristic features of the $\mathrm{Fe}$ growth on $\mathrm{Cu}(111)$ are its fcc structure inherited from the copper ${ }^{8-11}$ and its tendency towards step decoration. ${ }^{12}$ Using this effect we have prepared fcc Fe stripes on a vicinal $\mathrm{Cu}(111)$ substrate, which has a miscut of $1.2^{\circ}$ and an average terrace width of about $10 \mathrm{~nm}$. Prior to film preparation the copper substrate was cleaned by $\mathrm{Ar}^{+}$sputtering and annealing cycles to $700 \mathrm{~K}$. The crystallographic quality and the cleanliness of the substrate were monitored by LEED and AES, respectively. The Fe stripes were prepared in the analysis chamber from an iron wire ( $5 N$ in purity) heated by $e$-beam bombardment. At a typical Fe evaporation rate of $0.2 \mathrm{ML} / \mathrm{min}$ the pressure increased from $5 \times 10^{-11}$ mbar to $1 \times 10^{-10}$ mbar. To suppress the interdiffusion the copper substrate was kept at $0{ }^{\circ} \mathrm{C}$ during deposition. ${ }^{13}$ Afterwards the sample was further cooled down to $170 \mathrm{~K}$ in order to avoid a temperature rise above $0{ }^{\circ} \mathrm{C}$ during transferring. MOKE measurements were carried out in the sample temperature range between 90 and $270 \mathrm{~K}$, and STM data were recorded at room temperature after the MOKE measurements.

Figure 1 is a STM topography image showing the typical morphology of $1 \mathrm{D} \mathrm{Fe}$ stripes on $\mathrm{Cu}(111)$ at nominal coverages of 0.3 ML [Fig. 1(a)] and 0.8 ML [Fig. 1(b)]. The Fe stripes are located on the upper terrace of the $\mathrm{Cu}(111)$ steps and aligned along the $\langle 011\rangle$ direction. The marked line profile in Fig. 1(a) indicates that at 0.3 ML the Fe stripes are divided into segments which are mainly one monolayer in height and about 10-20 nm in average length (the dashed line stands for the height level of the substrate). The presence of a small fraction of bilayer high segments (marked as 2) is due to both the fact that the stripes at the edges of the wider terraces tend to collect more Fe adatoms and the fact that some copper diffuses onto the top of the Fe stripes during the STM experiments. The diffusion of the copper leaves some one-monolayer-deep holes in the regions between the $\mathrm{Fe}$ stripes. This finding is consistent with a previous STM study of the $\mathrm{Fe} / \mathrm{Cu}(111)$ system. ${ }^{9}$ However, it is worthwhile mentioning here that our magnetic measurements were performed on samples without any significant interdiffusion. At higher thickness of $0.8 \mathrm{ML}$ in Fig. 1(b), the Fe stripes become much more continuous as indicated by the marked line profile. The edges of the stripes are rough due to the tendency of the Fe edge atoms aligning along all three $\langle 011\rangle$ directions. The edge roughness together with some defects may cause some structural discontinuity along the stripes as shown in the line profile. 

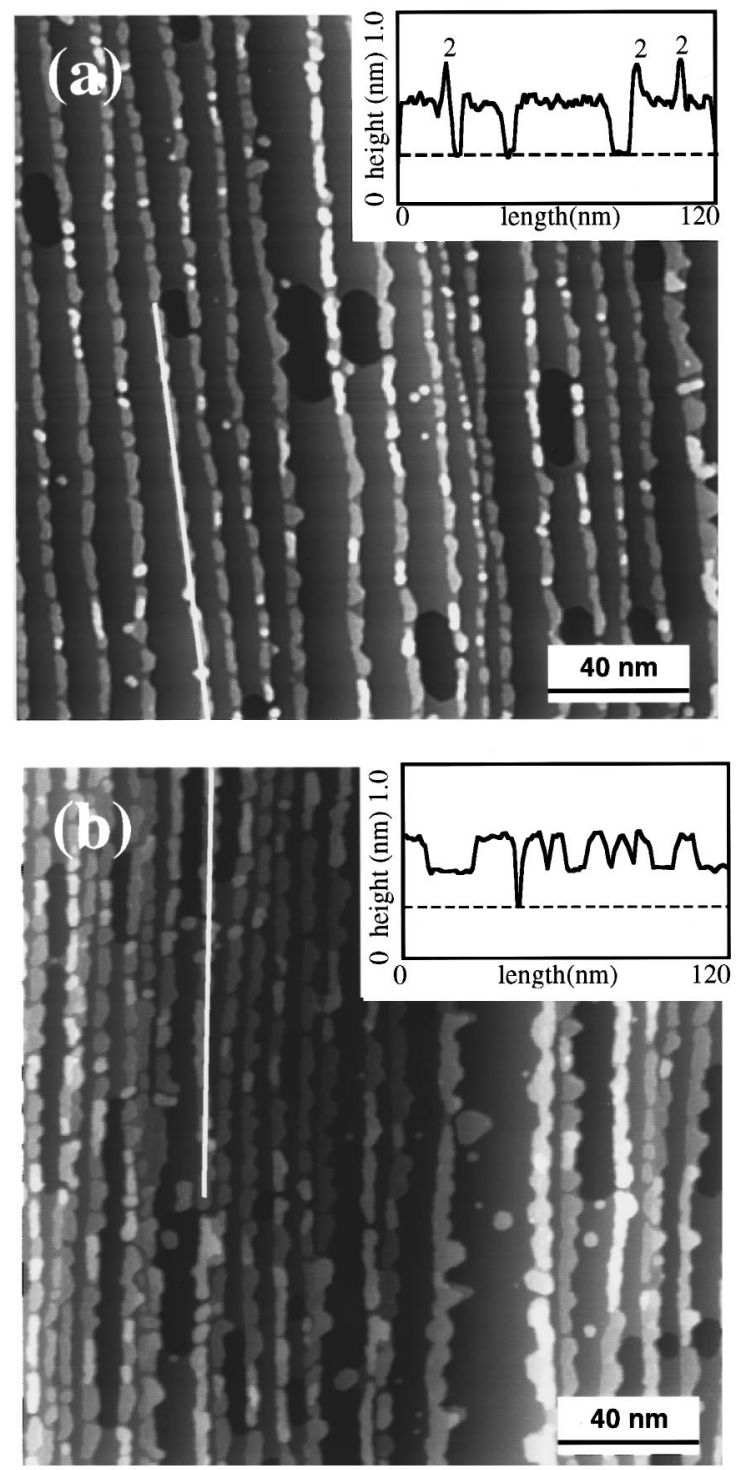

FIG. 1. STM topography image of 0.3-ML (a) and 0.8-ML (b) Fe stripes on $\mathrm{Cu}(111)$ vicinal surface. The marked line profiles are shown in the upper-right corner with the dashed lines indicating the height level of the substrate.

The MOKE measurements show that these Fe stripes exhibit hysteresis with a coercivity depending on the temperature. Figure 2(a) displays magnetic hysteresis loops for a 0.8 -ML film measured in the polar geometry. No magnetic signal was observed in the longitudinal geometry, irrespective of the external field being parallel or perpendicular to the stripes, indicating that the easy magnetization axis is along the surface normal. Figure 2(a) qualitatively shows that with increasing temperature the saturation magnetization $\left(M_{s}\right)$ is rather stable as compared to the quick decay of the remanent magnetization $\left(M_{r}\right)$. This is quantitatively demonstrated in Fig. 2(b), which displays the temperature dependence of $M_{s}$ and $M_{r}$ of the Fe stripes. Note in this case it is $M_{s}$ rather than $M_{r}$ that roughly follows the two-dimensional Ising model (the solid line in $M_{s}$ curve). Such phenomena have already been observed and discussed in a previous paper on a system of a monolayer of $\mathrm{Co}$ on $\mathrm{Cu}(111) \cdot{ }^{14}$ Upon a further increase of the temperature above $250 \mathrm{~K}$ the saturation drops down quickly, indicating that the system becomes nonmagnetic.
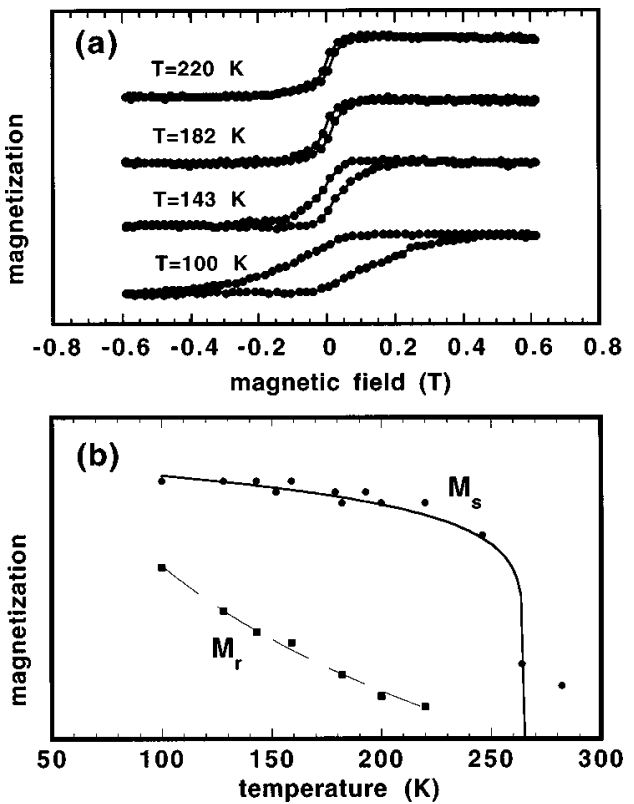

FIG. 2. (a) Magnetic hysteresis of a 0.8-ML Fe film measured at different temperatures. (b) Saturation and remanent magnetization plotted as a function of temperature. The solid lines are only for guiding the eye.

The magnetization of the Fe stripes is not only temperature but also time dependent. Figure 3 shows the time dependence of the magnetization of the 0.8-ML stripes. The zero level refers to the demagnetized state of the Fe stripes. After switching on the field $(\sim 0.6 \mathrm{~T})$ the magnetization of the stripes quickly saturates. When the field is removed, at low temperature of about $100 \mathrm{~K}$ the magnetization drops rapidly to about $70 \%$ of the $M_{s}$ and then it decays slowly. The slow decay of the magnetization at $100 \mathrm{~K}$ guarantees the ferromagnetic behavior of the system in any practical measuring time. At higher temperature of about $160 \mathrm{~K}$ the magnetization relaxation process becomes significantly faster. It is in-

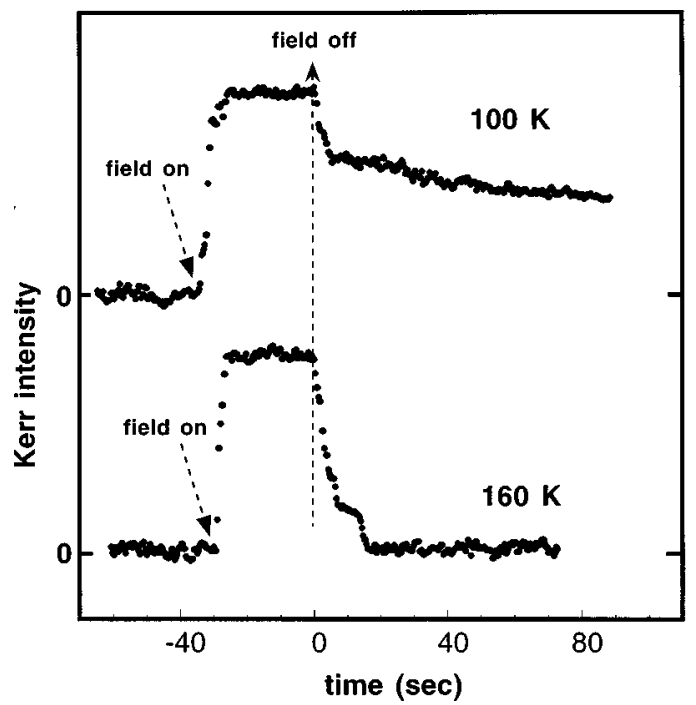

FIG. 3. Time dependence of the magnetization of a 0.8-ML film. After switching on the field (about $0.6 \mathrm{~T}$ ), the magnetization quickly saturates. About $30 \mathrm{sec}$ later the external field has been removed leading to the magnetization decaying slowly at low temperature $(100 \mathrm{~K})$ but very quickly at elevated temperature $(160 \mathrm{~K})$. 
teresting to note that the magnetization of the stripes with shorter segments (i.e., 0.3 ML) decays to zero rapidly at even much lower temperature of $50 \mathrm{~K}$.

We explain the magnetic behavior in terms of a model involving interacting Ising block spins. In particular, the measured magnetization hysteresis loops have been fitted by the suggested model, which yields fitting parameters such as the anisotropy constant $K_{1}$ and the volume of the block spin units. Although an Ising chain (or any anisotropic chain) is nonmagnetic in equilibrium, the validity of this result is restricted to a single stripe and the absence of magnetostatic interactions. In a real system which consists of a large number of parallel stripes, there is a coupling between neighboring stripes caused by the magnetostatic and RKKY interactions. Compared to the in-plane magnetized $\mathrm{Fe} / \mathrm{W}(110)$ stripes, ${ }^{6}$ the magnetostatic interaction could be much more important in the perpendicular magnetized $\mathrm{Fe} / \mathrm{Cu}(111)$ stripes. The minimization of the total magnetostatic energy would lead to an antiferromagnetic interaction between the neighboring stripes. The strength of the magnetostatic interaction is given by $H_{0}=(1 / 4 \pi) M_{s} t \int\left(1 / r^{3}\right) d A$, where $M_{s}$ is the spontaneous magnetization and $t$ is the thickness. ${ }^{15}$ A short calculation yields a simplified form, $H_{0}=\pi w t M_{s} /$ $8 \lambda^{2}$, where $w$ is the stripe width and $\lambda$ is the distance between the neighboring stripes. Taking the typical values $w=5 \mathrm{~nm}, t=0.2 \mathrm{~nm}, \lambda=10 \mathrm{~nm}$, and literature data $\mu_{0} M_{s}=0.75 \mathrm{~T},{ }^{16}$ we estimate that the magnetostatic interaction is of the order of $2 \mathrm{mT}$. This value is much smaller than the coercivity of the measured hysteresis loop, about $100 \mathrm{mT}$ at $100 \mathrm{~K}$, and is invisible in the measured hysteresis loops.

A more difficult problem is the RKKY-type interaction mediated by the substrate. Taking a typical coupling strength of the order of $0.4 \mathrm{~mJ} / \mathrm{m}^{2}$ at a copper interlayer distance of $1.3 \mathrm{~nm}$ (Ref. 17) and rescaling this value according to the $1 / r^{2}$ distance dependence for coupled layers yields interaction fields comparable to those caused by the magnetostatic interaction. The total coupling strength is much lower, since the RKKY interaction involves functions with oscillatory periods not exceeding a few tenths of a nanometer, whereas the scale of the morphological inhomogeneities clearly exceeds a few nanometers. In other words, due to the nonideal, patchy shape of the stripes and the slightly varying distance between the stripes, the positive and negative contributions are largely canceled out. Therefore, the RKKY interaction in the quasione-dimensional $\mathrm{Fe} / \mathrm{Cu}(111)$ films is unlikely to play an important role.

Another possible coupling mechanism between the stripes is magnetic quantum tunneling. But the temperature where the magnetic tunneling is effective has been observed below $10 \mathrm{~K}$, and in the tunneling regime the magnetization relaxation should be temperature independent. ${ }^{18-20}$ Since the decaying rate of the magnetization of the Fe stripes is strongly temperature dependent, the magnetization relaxation appears to be a thermally activated process rather than the magnetic quantum tunneling.

If the interaction between the stripes is negligible, the equilibrium of the system is superparamagnetic. There are interacting and therefore correlated block spins having parallel spin alignments inside each block, but due to thermal activation there is no long-range ferromagnetic order. The fact that the magnetization curves of the Fe films show hys-

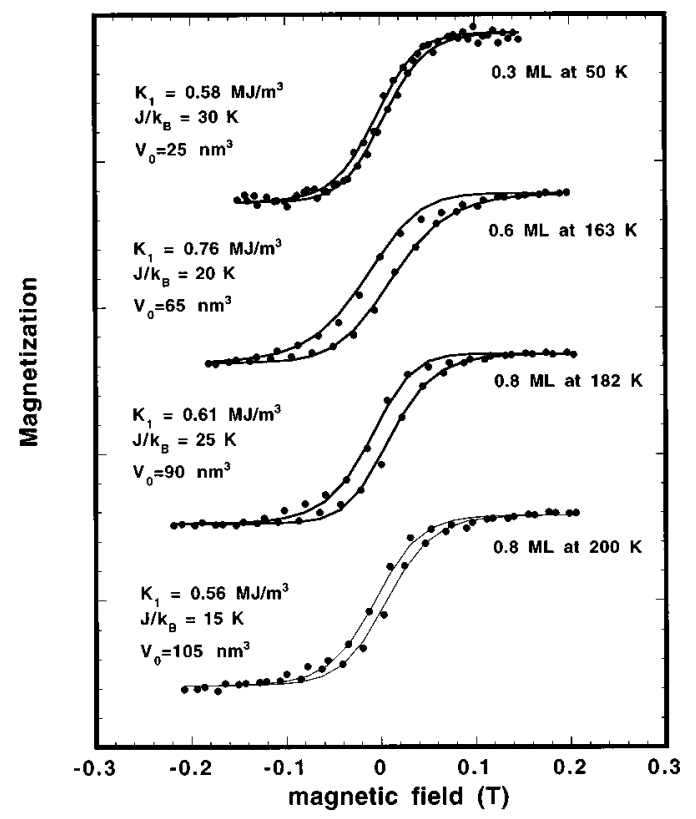

FIG. 4. Theoretical hysteresis curve (solid line) from Eq. (4) fitted to experimental data (full circles) from the stripes of $0.3 \mathrm{ML}$ at $50 \mathrm{~K}, 0.6 \mathrm{ML}$ at $163 \mathrm{~K}, 0.8 \mathrm{ML}$ at 182 and $200 \mathrm{~K}$, respectively.

teresis indicates that the films are in a frozen or blocked superparamagnetic state. The blocking temperature $T_{b}$ can be estimated from the vanishing of the coercivity, which is about $200 \mathrm{~K}$ for the $0.8-\mathrm{ML}$ film. Above $T_{b}$ the system behaves like a typical superparamagnet exhibiting no hysteresis but having essentially the same $M_{s}$ in an external field. At even higher temperatures, above about $270 \mathrm{~K}$, the individual spin blocks become magnetically disordered as indicated by the vanishing of $M_{s}$ [Fig. 2(b)].

To calculate the equilibrium magnetization of the $\mathrm{Fe}$ stripes, we generalize the one-dimensional Ising model to complex block spins. We start from the one-dimensional Ising model and obtain ${ }^{21}$

$$
\frac{M}{M_{s}}=\frac{\sinh \left(h / k_{B} T\right)}{\sqrt{\sinh ^{2}\left(h / k_{B} T\right)+\exp \left(-4 J / k_{B} T\right)}} .
$$

Here $h=\mu_{0} M_{s} H V_{0}$, where $H$ is the external field, $V_{0}$ is the volume of the block-spin segments, and $J$ is the exchange coupling between the segments. If there was no hysteresis, Eq. (1) could be used to fit the experimental magnetization curves. This fails, however, since Fig. 2 shows that the hysteresis loops are not restricted to very low temperatures. Thus, to achieve a comprehensive description of the stripes it is necessary to include nonequilibrium effects. The starting point of the consideration of nonequilibrium superparamagnetism is the master equation ${ }^{22}$

$$
\frac{d P\left(s_{Z_{i}}\right)}{d t}=W\left(-s_{Z_{i}} \rightarrow s_{Z_{i}}\right) P\left(-s_{Z_{i}}\right)-W\left(s_{Z_{i}} \rightarrow-s_{Z_{i}}\right) P\left(s_{Z_{i}}\right),
$$

where $s_{Z_{i}}= \pm 1$ indicate whether the magnetization of the $i$ th block-spin segment points in the $+\mathbf{e}_{z}$ or $-\mathbf{e}_{z}$ directions, respectively. $P\left(s_{Z_{i}}\right)$ and $W\left(s_{Z_{i}} \rightarrow-s_{Z_{i}}\right)$ denote probabilities and transition rates, respectively. The Ising model has no inherent dynamics, so that we choose $\mathrm{e}^{22,23}$ 


$$
\begin{aligned}
W\left(s_{Z_{i}} \rightarrow-s_{Z_{i}}\right)= & \frac{\Gamma_{0}}{2} \exp \left(-\frac{V_{0} K_{1}}{k_{B} T}\right) \\
& \times\left(1-s_{Z_{i}} \tanh \frac{h+J s_{Z_{i+1}}+J s_{Z_{i-1}}}{k_{B} T}\right),
\end{aligned}
$$

where the attempt frequency $\Gamma_{0}$ is of order $10^{9}-10^{12} \mathrm{~s}^{-1}$ (see, e.g., Ref. 24). After some calculation we obtain for small $J$ and narrow hysteresis loops

$$
\begin{aligned}
\frac{M}{M_{s}}= & {\left[1+\frac{2 J}{k_{B} T}\left(1-\tanh ^{2} \frac{h}{k_{B} T}\right)\right] } \\
& \times \tanh \frac{h}{k_{B} T}-\frac{r}{\Gamma}\left(1-\tanh ^{2} \frac{h}{k_{B} T}\right) \\
& \times\left[1-\frac{4 J r}{\Gamma k_{B} T}\left(1-4 \tanh ^{2} \frac{J}{k_{B} T}\right)\right],
\end{aligned}
$$

where $\Gamma=\Gamma_{0} \exp \left(-V_{0} K_{1} / k_{B} T\right)$ and $r=\left(1 / M_{s}\right)(\partial H / \partial t)$. In agreement with Fig. 3, it has been observed that larger sweeping rates $r$ lead to wider loops. Using Eq. (4) we have fitted four typical hysteresis loops of $0.3 \mathrm{ML}$ at $50 \mathrm{~K}, 0.6 \mathrm{ML}$ at $163 \mathrm{~K}, 0.8 \mathrm{ML}$ at $182 \mathrm{~K}$ and $200 \mathrm{~K}$, respectively. For all four curves the sweeping rate $r$ is about $0.04 \mathrm{~T} / \mathrm{s}$. The fitted curves as well as the fitting parameters $\left(K_{1}, J\right.$, and $\left.V_{0}\right)$ are shown in Fig. 4. The anisotropy constant $K_{1}$ is in the range between 0.55 and $0.80 \mathrm{MJ} / \mathrm{m}^{3}$. These values are an order of magnitude larger than the bulk anisotropy of $\alpha$-Fe $\left(0.05 \mathrm{MJ} / \mathrm{m}^{3}\right)$, indicating that the surface anisotropy is the dominant contribution to $K_{1}$. The fitted $V_{0}$ increases with increasing thickness, which is consistent with the STM images (see Fig. 1) showing that the segments in the stripes become longer with increasing thickness. Moreover, $V_{0}$ is

*Corresponding author.

${ }^{1}$ R. P. Erickson and D. L. Mills, Phys. Rev. B 43, 11527 (1991).

${ }^{2}$ U. Gradmann, J. Magn. Magn. Mater. 54-57, 733 (1986).

${ }^{3}$ G. F. Newell and E. W. Montroll, Rev. Mod. Phys. 25, 159 (1953); 25, 353 (1953).

${ }^{4}$ E. Ising, Z. Phys. 31, 253 (1925).

${ }^{5}$ L. J. de Jongh and A. R. Miedema, Adv. Phys. 23, 1 (1974); M. Steiner, J. Villain, and C. G. Windsor, ibid. 25, 87 (1975).

${ }^{6}$ H. J. Elmers, J. Hauschild, H. Höche, U. Gradmann, H. Bethge, D. Heuer, and U. Köhler, Phys. Rev. Lett. 73, 898 (1994).

${ }^{7}$ I. S. Jacobs and C. P. Bean, in Magnetism, edited by George T. Rado and Harry Suhl (Academic Press, New York, 1963), Vol. III, p. 300.

${ }^{8}$ U. Gradmann and P. Tillmanns, Phys. Status Solidi A 44, 539 (1977).

${ }^{9}$ Y. Darici, J. Marcano, H. Min, and P. A. Montano, Surf. Sci. 195, 566 (1988).

${ }^{10}$ D. Tian, F. Jona, and P. M. Marcus, Phys. Rev. B 45, 11216 (1992).

${ }^{11}$ M. T. Kief and W. F. Egelhof, Jr., J. Vac. Sci. Technol. A 11, 1661 (1993).

${ }^{12}$ A. Brodde, K. Dreps, J. Binder, Ch. Lunau, and H. Neddermeyer, Phys. Rev. B 47, 6609 (1993).

${ }^{13}$ M. Klaua, H. Höche, H. Jenniches, J. Barthel, and J. Kirschner, Surf. Sci. (to be published). also compatible to the actual geometry of the segments. Taking the average width of $4 \mathrm{~nm}$ and height of $0.2 \mathrm{~nm}$, for the 0.3 -ML stripes $\left(V_{0}=25 \mathrm{~nm}^{3}\right)$ we estimate the length of the block-spin unit to about $30 \mathrm{~nm}$. This agrees, as shown by the line profile in Fig. 1(a), well with the average length of the segments in the stripes.

Therefore, the fitting of Fig. 4 shows that the model gives a fair description of the magnetism of the stripes. The fact that $J$ has a nonzero value indicates the Fe stripes are different from a system consisting of isolated clusters or islands, whose $J$ should have zero value. The restriction of the model to small $J$ is reasonable for not too large coverages. Physically, the smallness of this value reflects the fact that the width of the stripes is not constant but has pronounced minima at which the switching of the magnetization is energetically favorable. There is, in fact, no major difficulty in generalizing Eq. (4) to large $J$, but then there may be other magnetization processes such as domain-wall motion along the stripes which are not included in the transition rates Eq. (3).

In conclusion we have prepared and investigated 1D fcc Fe stripes on $\mathrm{Cu}(111)$. The stripes are characterized by a perpendicular anisotropy of order of $0.6 \mathrm{MJ} / \mathrm{m}^{3}$ and exhibit a pronounced temperature and time dependence of the magnetization. The magnetic behavior of the stripes is reproduced by a micromagnetic generalization of the Ising model, which considers coupled block-spin segments. The nonequilibrium behavior of the stripes, that is the magnetic hysteresis, is explained by Glauber block-spin transitions.

The authors are grateful to Professor U. Gradmann for helpful discussions. We also acknowledge F. Pabisch, G. Kroder, and J. Barthel for technical support.

${ }^{14}$ J. Kohlhepp, H. J. Elmers, S. Cordes, and U. Gradmann, Phys. Rev. B 45, 12287 (1992).

${ }^{15}$ There is a logarithmically diverging self-energy contribution in this expression, which describes the interaction inside the stripes but does not contribute to the interaction between stripes.

${ }^{16}$ U. Gradmann, W. Kümmerle, and P. Tilmans, Thin Solid Films 34, 249 (1976).

${ }^{17}$ M. T. Johnson, S. T. Purcell, N. W. E. McGree, R. Coehoorn, J.aan de Stegge, and W. Hoving, Phys. Rev. Lett. 68, 2688 (1992).

${ }^{18}$ Eugene M. Chudnovsky, J. Appl. Phys. 73, 6697 (1993).

${ }^{19}$ J. Tejada, X. X. Zhang, and LI. Balcells, J. Appl. Phys. 73, 6709 (1993).

${ }^{20}$ B. Barbara, L. C. Sampaio, J. E. Wegrowe, B. A. Ratnam, A. Marchand, C. Paulsen, M. A. Novak, J. L. Tholence, M. Uehara, and D. Fruchart, J. Appl. Phys. 73, 6703 (1993).

${ }^{21}$ J. M. Yeomans, Statistical Mechanics of Phase Transitions (University Press, Oxford, 1992).

${ }^{22}$ K.-H. Fischer and A. J. Hertz, Spin Glasses (Cambridge University Press, Cambridge, England, 1991).

${ }^{23}$ Except the anisotropy prefactor, which is necessary to describe the perpendicular anisotropy of the film, Eq. (3) gives the wellknown Glauber transition rates.

${ }^{24}$ P. Gaunt, J. Appl. Phys. 59, 4129 (1986). 